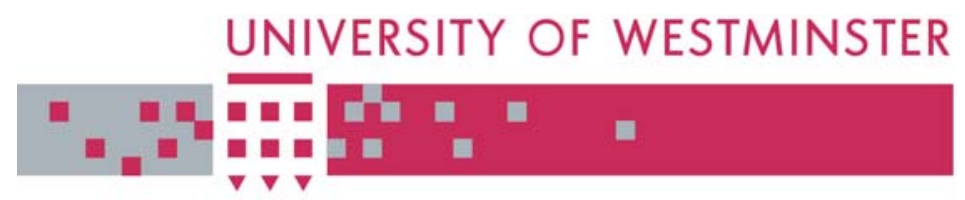

WestminsterResearch

http://www.wmin.ac.uk/westminsterresearch

\title{
Multi-dimensional matched filter identification technique for channel equalization deployed in spatial diversity receivers.
}

Adem Coskun

Izzet Kale

School of Electronics and Computer Science

Copyright (c) [2009] IEEE. Reprinted from PRIME: 2009 PhD Research in Microelectronics and Electronics. Proceedings. Cork, Ireland, 12-17 July 2009. IEEE, pp. 36-39. ISBN 9781424437337.

This material is posted here with permission of the IEEE. Such permission of the IEEE does not in any way imply IEEE endorsement of any of the University of Westminster's products or services. Personal use of this material is permitted. However, permission to reprint/republish this material for advertising or promotional purposes or for creating new collective works for resale or redistribution to servers or lists, or to reuse any copyrighted component of this work in other works must be obtained from the IEEE. By choosing to view this document, you agree to all provisions of the copyright laws protecting it.

The WestminsterResearch online digital archive at the University of Westminster aims to make the research output of the University available to a wider audience. Copyright and Moral Rights remain with the authors and/or copyright owners.

Users are permitted to download and/or print one copy for non-commercial private study or research. Further distribution and any use of material from within this archive for profit-making enterprises or for commercial gain is strictly forbidden.

Whilst further distribution of specific materials from within this archive is forbidden, you may freely distribute the URL of the University of Westminster Eprints (http://www.wmin.ac.uk/westminsterresearch).

In case of abuse or copyright appearing without permission e-mail wattsn@wmin.ac.uk. 


\title{
Multi-Dimensional Matched Filter Identification Technique for Channel Equalization deployed in Spatial Diversity Receivers
}

\author{
Adem Coskun and Izzet Kale \\ Applied DSP and VLSI Research Group \\ University of Westminster \\ London, W1W 6UW, United Kingdom \\ adem@alptron.com,kalei@westminster.ac.uk
}

\begin{abstract}
This paper proposes a multi-dimensional matched filtering technique for spatial diversity receivers. The coefficients of the multi-dimensional matched filter are identified by making use of an adaptive filter, the update of which doesn't require the transmission of any training symbols within the transmitted data stream. Therefore the use of the proposed technique improves the data rate efficiency. Furthermore, it is well known that implementing multi-dimensional matched filtering is essential for equalization purposes to obtain the optimum error rate performance from spatial diversity receivers. For that reason the technique is designed not only to identify the unknown matched filter but also to simultaneously lead to the equalization of the channel too. In order to update the adaptive filter, the Constant Modulus Algorithm (CMA) is utilized, which is an implementation convenient algorithm. Therefore the proposed technique is not computationally complex in comparison to those identification algorithms proposed for spatial diversity receivers. Simulations are provided to present the equalization performance of the novel technique.
\end{abstract}

\section{INTRODUCTION}

$\mathrm{T}$ HE use of spatial diversity, to improve the error rate performance, is one of the promising solutions for the reliability problems of wireless communication systems. Placing sufficiently spaced antennas at the receiver end would generate several independent channels and therefore enable reception of replicas of the transmitted signal. The channel of such a scheme of spatial diversity is called a Single Input Multi Output (SIMO) channel.

Many of the techniques used to establish the coefficients of a SIMO channel need the transmission of training signals in order to inform the receiver of the channel. This approach is inherently inefficient and wastes the bandwidth of the communication channel. That's why a blind scheme, which doesn't need any training period during the communication time, would definitely improve the system efficiency.

The blind methods, proposed for spatial diversity receivers, involve matrix decompositions, which are computationally expensive, such as Singular Value Decomposition (SVD) [1] and Eigen Value Decomposition (EVD) [2], [3]. In [4], Ozcelik et. al. proposed an alternative way to establishing the matched filter equivalent of a Single Input Single Output (SISO) channel, which stands for a communication system of single antennas at both transmitter and receiver. Due to the use of a computationally simple algorithm (i.e. CMA [5]) the proposed method did not require any matrix decomposition operation. In combination with a Decision Feedback Equalizer (DFE), it also equalized the channel. In this way; the Signal to Noise Ratio (SNR) is maximized, Inter Symbol Interference (ISI) is mitigated and the additive noise is whitened. Furthermore, for the calculation of the filter parameters of the DFE, only Second Order Statistics (SOS) of the unknown channel were used, which means more complicated statistics were not needed.

The optimal receiver strategy in terms of error rate for SIMO channels is to perform data symbol detection following Multi-dimensional Matched Filtering [6], which corresponds to performing matched filtering at every single antenna simultaneously. Therefore, the adaptation of the receiver in [4] for SIMO channels would both improve the efficiency and performance in addition to its computational cost.

For the SIMO communication model given in Fig. 1, where the DFE equalization is performed following a multi dimensional matched filter, the same method as in [4] can be used for each channel; however such an operation will not improve the Bit Error Rate (BER) performance and would not provide any gain from the diversity of using multiple antennas. Thus, a more effective technique has been devised and implemented in this paper. To do so, the DFE and the blind matched filter blocks have been adapted for the SIMO case, which will be presented in details in the next section. The last section of the paper presents, simulation results of the equalization and estimation performance for the novel technique.

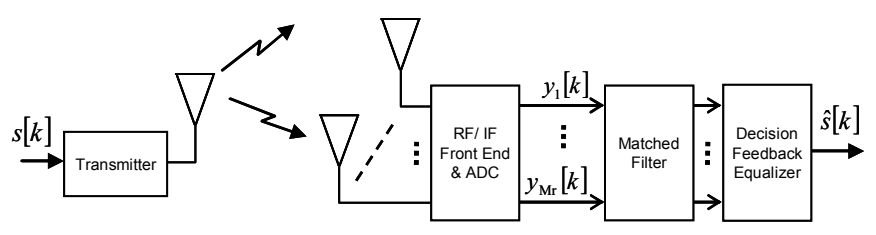

Fig. 1. Block diagram of the system 


\section{System Model AND Novel SIMO ReCeIVER}

\section{A. System Model}

We have assumed a single transmitter/receiver scenario, where $M_{r}$ antennas are placed at the receiver while the transmitter has a single antenna (i.e. $\mathrm{M}_{\mathrm{t}}=1$ ). Hence, in total there are $\mathrm{M}_{\mathrm{r}}$ channels, through which the signal transmission is conducted. The channels in between each receiving antenna and the transmitter are frequency selective and time invariant. Each of the $\mathrm{M}_{\mathrm{r}}$ frequency selective channels is assumed to be composed of $\mathrm{L}$ discrete coefficients. The channel for any one of the $\mathrm{M}_{\mathrm{r}}$ receive antenna can be written as;

$$
\mathbf{y}_{\mathrm{i}}[k]=\mathbf{H}_{\mathrm{i}} \mathbf{s}[k]+\mathbf{n}_{\mathrm{i}}[k] \quad \text { for } \mathrm{i}=1, \cdots, \mathbf{M}_{\mathrm{r}}
$$

where $\mathbf{y}_{\mathrm{i}}[k]=\left[y_{\mathrm{i}}[k] \cdots \quad y_{\mathrm{i}}[k+\mathrm{T}-1]\right]$ is the stacked vector of received signals and $\mathbf{n}_{i}$ is the stacked vector of noise components for the $i^{\text {th }}$ receive antenna. Both vectors $\mathbf{y}_{\mathrm{i}}$ and $\mathbf{n}_{\mathrm{i}}$ are of length $T x 1$ (corresponds to $T$ times one), where $T$ represents the number of taps of the equalizer placed after a receive antenna. Noise is assumed to be Additive White Gaussian (AWGN) and $\mathbf{s}$ is the vector of complex data symbols, denoted by $s[k]$. The channel matrix $\mathbf{H}_{\mathrm{i}}$ is in the form as defined in (2) below;

$$
\mathbf{H}_{\mathrm{i}}=\left[\begin{array}{cccccc}
h_{\mathrm{i}}[\mathrm{L}-1] & \cdots & h_{\mathrm{i}}[0] & 0 & \cdots & 0 \\
\vdots & \ddots & \ddots & \ddots & \ddots & \vdots \\
0 & \cdots & 0 & h_{\mathrm{i}}[\mathrm{L}-1] & \cdots & h_{\mathrm{i}}[0]
\end{array}\right]
$$

Each row of $\mathbf{H}_{\mathrm{i}}$ contains the multipath components of the $\mathrm{i}^{\text {th }}$ channel that is $\mathbf{h}_{\mathrm{i}}=\left\{h_{\mathrm{i}}[\mathrm{L}-1] \quad \cdots \quad h_{\mathrm{i}}[0]\right\} . y_{\mathrm{i}}[k]$ denotes the data symbol received at time $k$ by antenna i [6, page 139].

\section{B. Novel SIMO Receiver Model}

The communication model in Fig. 1 can be extended as in Fig. 2, where the channel of each receive antenna is matched filtered and subsequently equalized using a linear DFE. The DFE is called "linear" since the Decision Device (DD) is placed after the feedback filter. This setup is preferred in order to maintain the linearity throughout the system.

Each matched filter, $\mathbf{m}_{\mathbf{i}}$, is the conjugated time-reversed version of its corresponding channel $\mathbf{h}_{\mathrm{i}}$, i.e. $\mathbf{m}_{\mathrm{i}}[k]=\mathbf{h}_{\mathrm{i}}^{*}[\mathrm{~L}-k]$. As can be seen from Fig. 2, every receive antenna needs a feedforward filter, $\mathbf{f}_{\mathrm{i}}$, while the single feedback filter $\mathbf{b}$ is enough to remove the post-Inter Symbol Interference from the signal, generated by adding the outputs of feedforward filters [7]. In Fig. 2, the block, which has the filter $\mathbf{b}$ at its feedback, is denoted by B. The delay, introduced to the symbol estimate $\hat{s}$ by the receiver blocks, is given by $\mathrm{D}$.

"Given a discrete-time network, a node with $\mathrm{N}$ incoming branches with Transmittances $T_{i 1}, T_{i 2}, \ldots, T_{i \mathrm{~N}}$ and $\mathrm{M}$ outgoing branches $T_{O 1}, T_{O 2}, \ldots, T_{O N}$ can be replaced by NxM branches with transmittances $T_{i 1} T_{O 1}, T_{i 1} T_{O 2}, \ldots, T_{i \mathrm{~N}} T_{O \mathrm{M}}$." [8]. If the adder in the DFE is regarded as the node, which is the junction of incoming and outgoing branches, then the sub-block B in Fig. 2 can be carried before the node as in Fig. 3, which will

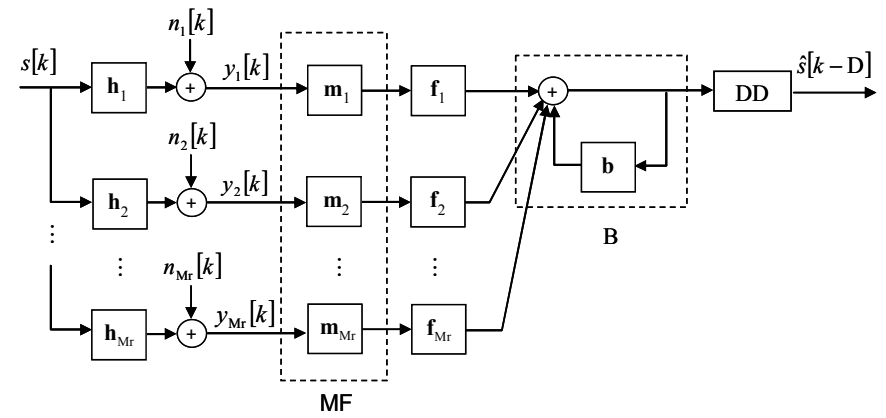

Fig. 2. SIMO-DFE receiver with multi-dimensional matched filtering

correspond to the same behavioral representation. In Fig. 3, maintaining the linearity, the multi-dimensional matched filter was placed following B.

As long as the order of the feedback filters are kept small, the complexity of the receiver would not be affected as a consequence of the use of several feedback filters. Moreover, the direct canonic realization of the discrete time network of the consecutive filters $\left(\mathbf{f}_{i}\right.$ and $\left.\mathrm{B}\right)$ at each receive branch yields a canonic structure, where it is possible to share the unit delays used for the feedback filter $\mathbf{b}$ with those of the feedforward structure. [8]

In [4], it has been shown that the weights of an adaptive filter, using the CMA, can effectively converge to the matched filter equivalent of the channel, if the matched filter itself is the sole unknown in the receiver. Building on [4], for blind multi-dimensional matched filtering; the CMA can also be utilized with the new technique for SIMO. For this purpose an adaptive filter, whose coefficients are updated over the use of the CMA, is placed instead of the multi-dimensional matched filters in Fig.3 and the novel architecture is plotted as in Fig. 4

By using the receiver in Fig. 4, the data symbols can be identified and the matched filter equivalent of the channels $\left(\mathbf{h}_{1}, \mathbf{h}_{2}, \cdots, \mathbf{h}_{\mathrm{Mr}}\right)$ can be found as the coefficients of the receiver sub-block, denoted by CMA-SIMO.

Adaptation of the novel blind DFE scheme will be explained in the next part.

\section{Decision Feedback Equalizer}

The optimal feedforward and feedback filter weights of the DFE for SIMO channels can be calculated by multiplying the correlation matrices $\mathbf{R}_{\mathrm{a}}$ and $\mathbf{R}_{\mathrm{b}}$ (i.e. $\widetilde{\mathbf{g}}=\mathbf{R}_{\mathrm{a}} \mathbf{R}_{\mathrm{b}}{ }^{-1}$ ), where the

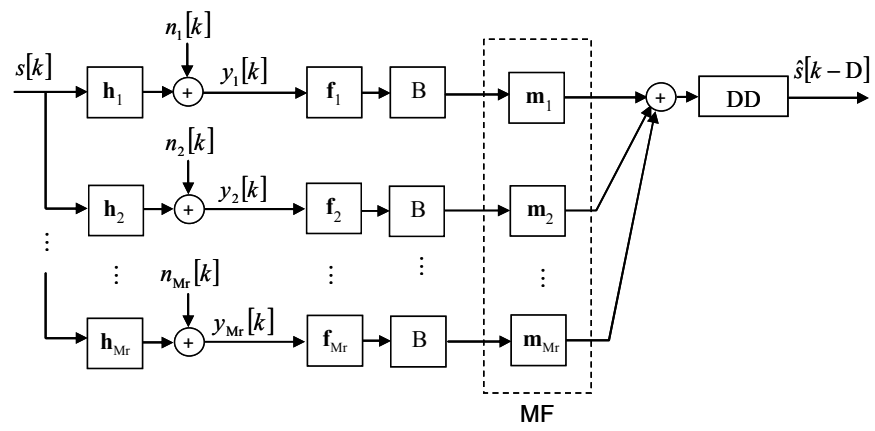

Fig. 3. Novel spatial diversity receiver 


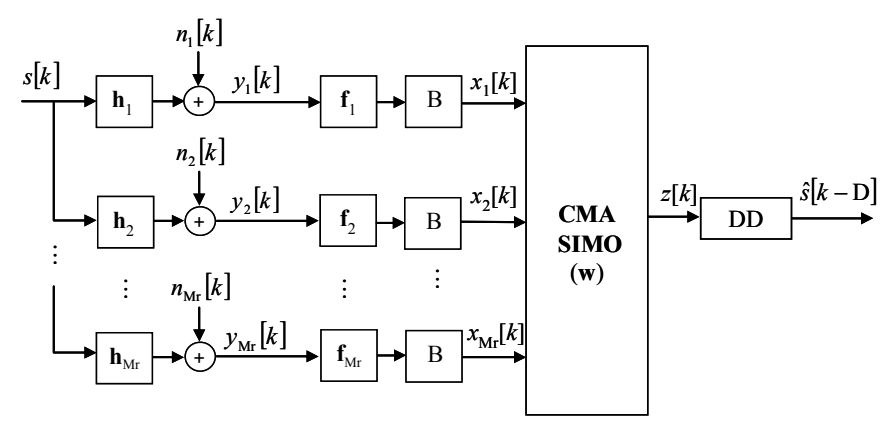

Fig. 4. Novel blind spatial diversity receiver

$1 \mathrm{x}\left(\mathrm{M}_{\mathrm{r}} \mathrm{T}+\mathrm{F}\right)$ sized vector $\widetilde{\mathbf{g}}=[\mathbf{f} ;-\mathbf{b}]$ is composed of concatenated feedforward filters (i.e. $\mathbf{f}=\left[\mathbf{f}_{1} \cdots \mathbf{f}_{\mathrm{Mr}}\right]$ ) of length $1 \mathrm{xM}_{\mathrm{r}} \mathrm{T}$ and the feedback filter of length $1 \mathrm{xF}$.

The more explicit description on $\mathbf{R}_{\mathrm{a}}$ and $\mathbf{R}_{\mathrm{b}}$ can be found in [6], but briefly we can say that their calculation needs the channel of every single antenna branch to be known. For our blind receiver the channels, effective over the DFE filter, are the "channel+matched filter" combinations as can be seen in Fig. 2. As long as the matched filter is the conjugate of the channel, reversed in time domain, the effective channel over the DFE of branch i can be written as;

$$
h_{\mathrm{i}}^{\text {eff }}[k]=\sum_{j=0}^{\mathrm{L}-1} h_{\mathrm{i}}[j] h_{\mathrm{i}}^{*}[(\mathrm{~L}-1)-k+j]
$$

It can be realized from (3) that the effective channel corresponds to the autocorrelation of the vector $\mathbf{h}_{\mathbf{i}}$, which may also be found blindly by correlating the channel output as;

$$
h_{\mathrm{i}}^{e f f}[k]=\sum_{j=0}^{\mathrm{N}} y_{\mathrm{i}}[j] y_{\mathrm{i}}^{*}[j+k] /\left(\mathrm{K} \sigma_{s}^{2}\right)
$$

since the transmitted data symbols $s[k]$ are i.i.d. [4]. The use of the formula that is given in (4) enables the calculation of the autocorrelation (and therefore the effective channel) by manipulating the channel output without the need for a priori channel information, where $\sigma_{\mathrm{s}}$ is the standard deviation of data symbols and $\mathrm{K}$ is the number of data symbols to be used for the estimation of the autocorrelation vector.

Once the DFE filter weights are obtained, which then leaves the matched filters as the only unknown in the system; it is guaranteed that the adaptive filter CMA-SIMO will converge to the matched filters for the unknown channels [4].

\section{Constant Modulus Algorithm}

CMA aims to update the coefficients of a filter for adaptive blind channel equalization purposes. Fig. 4 shows that the multi-dimensional matched filter is substituted with the CMASIMO block, where $\mathbf{w}$ represents the vector of filter coefficients to be estimated adaptively by the CMA.

The criterion for CMA type filter adaptation is to minimize the Constant Modulus (CM) cost function $J(\mathbf{w})$, defined by $J(\mathbf{w})=1 / 4 E\left\{\left(|z[n]|^{2}-R_{2}\right)^{2}\right\}$, where the dispersion constant $R_{2}$ is $E\left\{|s[k]|^{4}\right\} / E\left\{|s[k]|^{2}\right\}$ and $E\{$.$\} denotes the$ statistical expectation operation. If a data symbol constellation, which has a constant squared-modulus of one (i.e. $|s[k]|^{2}=1$ ), is used then $R_{2}$ will also be equal to one. Using stochastic gradient descent, the CMA-SIMO vector coefficients can be updated by deploying the following formulation [9];

$$
\mathbf{w}(n+1)=\mathbf{w}(n)+\mu \mathbf{x}[k] z[n]\left(|z[n]|^{2}-1\right)
$$

where $n$ represents the iteration number. $\mathbf{w}(n)$ is the CMASIMO vector at the $n^{\text {th }}$ iteration and similarly z $[n]$ is the output of the CMA-SIMO block at the $n^{\text {th }}$ iteration. $\mu$ is a small positive step size and $\mathrm{x}[k]$ can be written as follows [9];

$$
\begin{aligned}
\mathbf{x}[k]=\left[x_{1}[k], x_{1}[k-1]\right. & , \cdots, x_{1}[k-L], \\
& \left.x_{2}[k], x_{2}[k-1], \cdots, x_{\mathrm{Mr}}[k-L]\right]
\end{aligned}
$$

\section{Simulations}

In order to understand how well the novel blind technique equalizes a SIMO channel, two sets of simulations have been plotted in Fig. 5. For the simulations, unit energy QPSK symbol transmission is assumed and the noise is AWGN. Rayleigh distributed SIMO channels have been tested and the BER average has been taken for each set of simulations. Therefore one should note that for individual channels, the performance plotted in Fig. 5 may vary; even for some channels far better results might be obtained like the four channels given in Table I and Table II. In these Tables, two types of channels are considered; Minimum-phase and NonMinimum phase.

The first case plotted in Fig. 5, is a 3-antenna receiver case, where each channel has 2 taps (i.e. $\mathrm{L}=2$ ). In the Fig. 5, the case was indicated by the notation "1x3 (2-taps)", where the first portion represents the number of antennas at the

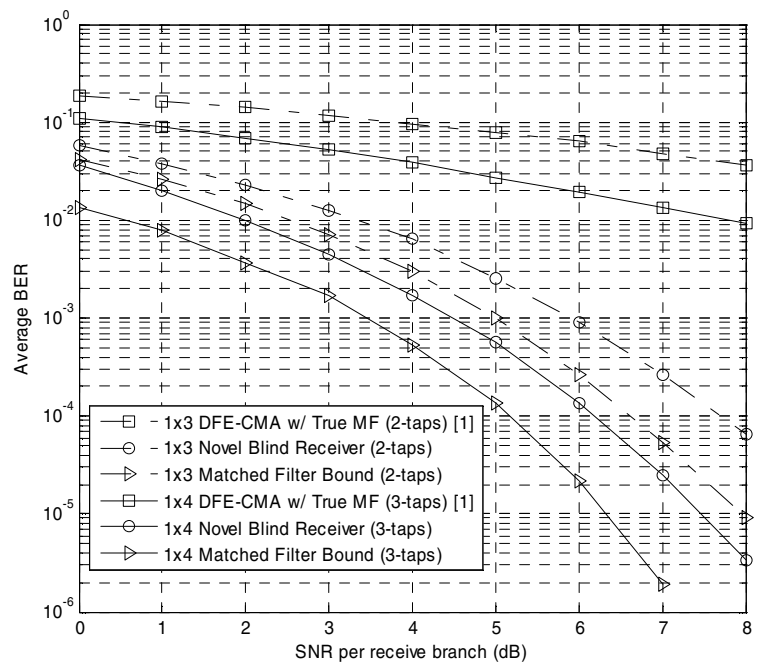

Fig. 5. BER comparison for the Novel Blind Scheme. The simulation parameters were chosen as; $\mathrm{T}=8$ (The size of each feed-forward filter of the $\mathrm{DFE}$ ), $\mathrm{F}=2$ (The size of feed-back filter of DFE), $\mathrm{D}=4$ (Delay), $\mu=0.01$ (Step size for the CMA). 
TABLE I

SET OF CHANNEL RESPONSES \# 1

\begin{tabular}{lcccccc}
\hline Channel Type & $h_{l}[1]$ & $h_{I}[2]$ & $h_{2}[1]$ & $h_{2}[2]$ & $h_{3}[1]$ & $h_{3}[2]$ \\
\hline \hline $\begin{array}{l}\text { Minimum Phase } \\
\begin{array}{l}\text { Non-Minimum } \\
\text { Phase }\end{array}\end{array}$ & -0.8249 & -0.5653 & 0.9737 & -0.2278 & 0.7171 & -0.6969 \\
\hline
\end{tabular}

TABLE II

SET OF CHANNEL RESPONSES \#2

\begin{tabular}{lccccccccccccc}
\hline Channel Type & $h_{1}[1]$ & $h_{l}[2]$ & $h_{1}[3]$ & $h_{2}[1]$ & $h_{2}[2]$ & $h_{2}[3]$ & $h_{3}[1]$ & $h_{3}[2]$ & $h_{3}[3]$ & $h_{4}[1]$ & $h_{4}[2]$ & $h_{4}[3]$ \\
\hline \hline $\begin{array}{l}\text { Minimum Phase } \\
\begin{array}{l}\text { Non-Minimum } \\
\text { Phase }\end{array}\end{array}$ & -0.7801 & -0.5345 & -0.3252 & 0.8189 & -0.1915 & -0.5410 & 0.5960 & -0.5792 & 0.5562 & 0.9444 & 0.1621 & 0.2861 \\
\hline
\end{tabular}

transmitter and receiver and the following portion in parenthesis gives the length of each channel realized from each receiving antenna. The first set of channel responses in Table I are of the first case. For the second case, an extra antenna was added to the system in addition to an increase in the length of the channel from 2 to 3 . The channel set in Table II belongs to this case.

The reason as to why we kept the size of the channel so short was to realize the diversity gain over the BER curve more easily. For channels with higher orders, our method converges without problems. In order to plot the matched filter bound, a single shot transmission is assumed [6] in order to realize the ISI-free performance of the system.

These plots reveal that the novel blind receiver performs equalization close to the matched filter bound. The diversity gain, for the $1 \times 3$ SIMO channel having 2 taps reaches up to 6 , which is the expected gain to be obtained from such a case. For the second system the increase in the diversity gain in addition to the array gain is realizable. It should also be mentioned that our novel technique has its shortcomings too. This is due to the matched filter coefficients, being found by blind estimation and thus in estimation error, which in high SNR values resulting a loss in the diversity gain for our novel technique. Furthermore, because a linear model for the DFE is preferred rather than that of a non-linear structure, the BER plot is further from the MF Bound.

The improvement obtained by the novel design is also compared with the previous work of [4]. The receiver in Fig. 3 can also be implemented by simply placing the receiver, proposed in [4], at every branch leading to an identical structure with the only difference being in the implementation algorithms. The notation "DFE-CMA" is used to indicate the results obtained from the aforementioned implementation. It should be noted that without the novel design the diversity gain can not be realized by placing a DFE-CMA combination of filter blocks at each receive branch.

The estimation error for the matched filters was also evaluated in the Mean Squared Error (MSE) sense, defined as $M S E=\left(\sum_{\mathrm{i}=1}^{\mathrm{M}_{\mathrm{r}}}\left\|\hat{\mathbf{m}}_{\mathrm{i}}-\mathbf{m}_{\mathrm{i}}\right\|_{2}^{2}\right)\left(\sum_{\mathrm{i}=1}^{\mathrm{M}_{\mathrm{r}}}\left\|\mathbf{m}_{\mathrm{i}}\right\|_{2}^{2}\right)^{-1}$, where $\hat{\mathbf{m}}_{\mathrm{i}}$ is the channel vector estimated by the novel method and $\mathbf{m}_{\mathrm{i}}$ is the vector of true channel coefficients. $\|\cdot\|_{2}$ represents the Euclidean norm operation. An average MSE rate of $-20.53 \mathrm{~dB}$ has been realized at $10 \mathrm{~dB}$ SNR for the $1 \times 3$ case having 2-taps after the SIMO-CMA vector, w, has been updated during $1 \times 10^{4}$ symbol periods.

\section{CONCLUDING REMARKS}

In this paper, we have presented a novel technique for multi-dimensional matched filtering deployed in spatial diversity receivers. Computer simulation results show that the novel blind structure is capable of identifying the matched filter of an unknown SIMO channel as well as blindly equalizing it. The expected diversity gain over SIMO channels is obtained by the novel approach, which outperformed the SISO counterpart [4]. Neither any decomposition operation was utilized nor statistics higher than second order are used for the implementation of our novel method; therefore it is computationally convenient and of lower complexity with respect to several approaches reported in the literature. The BER performance is shown to be close to the MF bound and therefore is preferable over non-blind schemes.

\section{REFERENCES}

[1] L. Tong, G. Xu, and T. Kailath, "Blind identification and equalization based on second-order statistics: A time domain approach," IEEE Trans. Information Theory, vol. 40, no. 2, pp. 340-349, Mar. 1994.

[2] G. Xu, H. Liu, L. Tong and T. Kailath, "A least squares approach to blind channel identification," IEEE Trans. Signal Processing, vol 43, pp. 2982-2993, Dec. 1995.

[3] E. Moulines, P. Duhamel, J.-F. Cardoso, and S. Mayrargue, "Subspace methods for the blind identification of multichannel FIR filters," IEEE Trans. Signal Processing, vol. 45. pp. 516-525, Feb. 1995.

[4] I. Ozcelik, I. Kale, B. Baykal, "Blind adaptation of a matched filter using the Constant Modulus Algorithm coupled with an optional correction method" in IEEE ISSPA'03, vol. 2, pp. 291-294, July 2003.

[5] D. Godard, "Self-recovering equalization and carrier tracking in twodimensional data communications systems," IEEE Trans. Commun., vol. COM-28, no. 10, pp. 1867-1875, 1980.

[6] A. Paulraj, R. Nabar and D. Gore, Introduction to Space-Time Wireless Communications, Cambridge University Press, 2005.

[7] J. K. Hwang, C. H. Chen and M. C. Tsai, "Design and analytical error performance of MMSE-DFE receiver with spatial diversity", in IEEE SPAWC'01, pp. 13 -16, Mar. 2001

[8] A. Antoniou, Digital Signal Processing: Signals, Systems, and Filters, McGraw-Hill, New York, 2006.

[9] G. Li, Z. Ding, "New blind/semi-blind equalization algorithms for GSM systems," in IEEE SPAWC '99, pp. 66-69, May 1999. 